\title{
Evaluation of temperature effects on brake wear particles using clustered heatmaps
}

\author{
Jihoon Shin ${ }^{1}$, Inhyeok Yim ${ }^{1}$, Soon-Bark Kwon ${ }^{2}$, Sechan Park ${ }^{3}$, Min-soo Kim ${ }^{4}$, YoonKyung Cha $^{{ }^{1+}}$ \\ ${ }^{1}$ Department of Environmental Engineering, University of Seoul, Seoul 02504, Republic of Korea \\ ${ }^{2}$ DAP Inc., 176 Cheoldobagmulgwan-ro, Uiwang 16105, Republic of Korea \\ ${ }^{3}$ Transportation System Engineering, University of Science and Technology (UST), Uiwang 16105, Republic of Korea \\ ${ }^{4}$ Urban Transit Research Team, Korea Railroad Research Institute (KRRI), Uiwang 16105, Republic of Korea
}

\begin{abstract}
Temperature effects on the generation of brake wear particles from railway vehicles were generated, with a particular focus on the generation of ultrafine particles. A real scale brake dynamometer test was repeated five times under low and high initial temperatures of brake discs, respectively, to obtain generalized results. Size distributions and temporal patterns of wear particles were analyzed through visualization using clustered heatmaps. Our results indicate that high initial temperature conditions promote the generation of ultrafine particles. While particle concentration peaked within the range of fine sized particles under both low and high initial temperature, an additional peak occurred within the range of ultrafine sized particles only under high initial temperature. The timing of peak occurrence also differed between low and high initial temperature conditions. Under low initial temperature fine sized particles were generated intensively at the latter end of braking, whereas under high initial temperature both fine and ultrafine particles were generated more dispersedly along the braking period. The clustered correlation heatmap divided particle sizes into two groups, within which generation timing and concentration of particles were similar. The cut-off point between the two groups was approximately $100 \mathrm{~nm}$, confirming that the governing mechanisms for the generation of fine particles and ultrafine particles are different.
\end{abstract}

Keywords: Brake wear particle, Clustered correlation heatmap, Clustered heatmap, Railway vehicle, Temperature, Ultrafine particle

\section{Introduction}

Particulate matter (PM), composed of a mixture of solid and liquid particles suspended in the air, is a widespread pollutant that has detrimental effects on respiratory health [1]. Although various sources produce PM, its primary sources in urban environment are traffic-related particles. Traffic-related particles are largely classified into exhaust particles created during the combustion process, and non-exhaust particles caused by factors other than combustion. Subsequent to the implementation of control programs, exhaust particle concentrations have gradually decreased, whereas less strict regulations on non-exhaust particles have increased their relative contribution to PM [2-4]. Non-exhaust particles mainly originate from brakes, tires, and road resuspensions, with brake wear particles comprising the largest proportion [5] and being the most important cause [6, 7]. Approximately 35\% of brake wear mass was reported to be emitted as atmospheric particles [8], and brake dynamometer tests showed that approximately $50 \%$ of wear debris becomes airborne [9].

Although the fact that small PM is more hazardous than large PM is well understood, studies on small-sized, non-exhaust particles from railway vehicles are rare $[10,11]$. Therefore, this study focused on identifying the characteristics of railway vehicle brake wear particles, in particular ultrafine particles $(\leq 0.1 \mu \mathrm{m})$. Existing studies reported that a number of factors, including speed or brake force, influence the generation of ultrafine particles [12-17]. When the high speed or brake force resulted in surface temperature exceeding a certain level, the generation of ultrafine particles increased. The critical temperature associated with increasing ultrafine particles differed by experimental methods, ranging from $\sim 70-300^{\circ} \mathrm{C}[12,13,18,19]$. In this regard, the experimental design of this study aimed to evaluate the temperature effects on the generation of ultrafine particles by differentiating initial temperature conditions. It was hypothesized that under high initial tem-
This is an Open Access article distributed under the terms of the Creative Commons Attribution Non-Commercial License (http://creativecommons.org/licenses/by-nc/3.0/) which permits unrestricted non-commercial use, distribution, and reproduction in any medium, provided the original work is properly cited.
Received November 1, 2018 Accepted January 12, 2019

${ }^{\dagger}$ Corresponding author

Email: ykcha@uos.ac.kr

Tel: +82-2-6490-2872 Fax: +82-2-6490-2859

Copyright (C) 2019 Korean Society of Environmental Engineers 
perature, the temperature would be more likely to reach a critical level during the braking process, leading to increases in the generation of ultrafine particles. Also, taking the stochastic nature of brake wear particle generation into account, this study conducted replicate experiments under each of two different temperature conditions to make generalizable inferences from data. As an analysis framework, we propose to use clustered heatmaps, which have been recently used in biological sciences and other fields, and were proved to effectively display multidimensional, hierarchical data in a single figure [20].

The objective of this study was to evaluate the temperature effects on brake wear particle from a railway vehicle using clustered heatmap. Fine and ultrafine sized particles in the range of 5.6 to $560 \mathrm{~nm}$ were collected and measured from five replicate tests using real scale brake dynamometer, and were analyzed through visualization using time series and correlation clustered heatmaps.

\section{Methods}

\subsection{Braking Tests}

Braking tests were performed to obtain particle emission data from railways. Braking tests were performed with a real scale brake dynamometer, and the components of the brake dynamometer, such as brake discs and non-asbestos organic (NAO) pads, were equipped referring to the UIC Code 541-3 international standard [21]. Initial velocity, braking force, and braking time, which were set at $60 \mathrm{~km} / \mathrm{h}, 8 \mathrm{kN}$, and $36 \mathrm{~s}$, respectively, approximated the operating conditions for actual high-speed railways. In order to characterize the variations in particle generation with temperature, we established low and high temperature categories, which corresponded to initial temperatures of 35 and $60^{\circ} \mathrm{C}$, respectively. Sliding surface temperature was measured with the contact type sensor. Six sensors were installed, with three on the front side of the brake disc and the other three on the back side of the brake disc. The temperature measurements from the six sensors were averaged to be used for analysis. Because particle generation during braking is a stochastic process, five replicate tests were performed under each temperature condition to obtain more generalized results [18]. Equipment operation and experimental condition changes were conducted remotely from a control room, and pollutants generated during the braking test process were purified with a ventilation unit.

A fast mobility particle sizer (FMPS, TSI 3091) was used to measure fine and ultrafine particles generated during the braking test, and the sampling probe was installed approximately $5 \mathrm{~cm}$ from the braking area. The FMPS determined the particle size by controlling the charging state of nanoparticles and measuring electrical mobility with current capacity. It also provided the particle size distribution data of 32 channels ranging in size from 5.6-560 nm. Details of the channels are shown in Table S1.

\subsection{Data Analysis}

\subsubsection{Data preprocessing}

A dataset was established from the braking tests and was subject to preprocessing for more efficient interdependence analysis. To account solely for particle concentrations that result from braking, the mean particle concentration during the $5 \mathrm{~s}$ prior to braking was subtracted from the measured particle concentration. In addition, the effects due to differences in the particle concentration ranges from each test were removed by normalization, whereby the data were adjusted to range from 0 to 1 . Furthermore, fine and ultrafine particles were categorized into two size classes, with ultrafine particles sizes $\leq 100 \mathrm{~nm}$ being marked as class 1 , and fine particles being marked as class 2 . Note that the class 1 corresponded to the size channels 1-20, and class 2 corresponded to the channels 21-32.

\subsubsection{Clustered heatmaps}

A clustered heatmap uses a color scale to shade values in a data matrix and displays the hierarchical cluster dendrograms of each row and column on the margins. Clustered heatmaps show data patterns by integrating information into a single figure and have been used recently in the biological sciences [20]. Four factors must be determined to generate a clustered heatmap. First, a preprocessing algorithm which can minimize noise is needed; in this study, a normalization algorithm was applied in the data

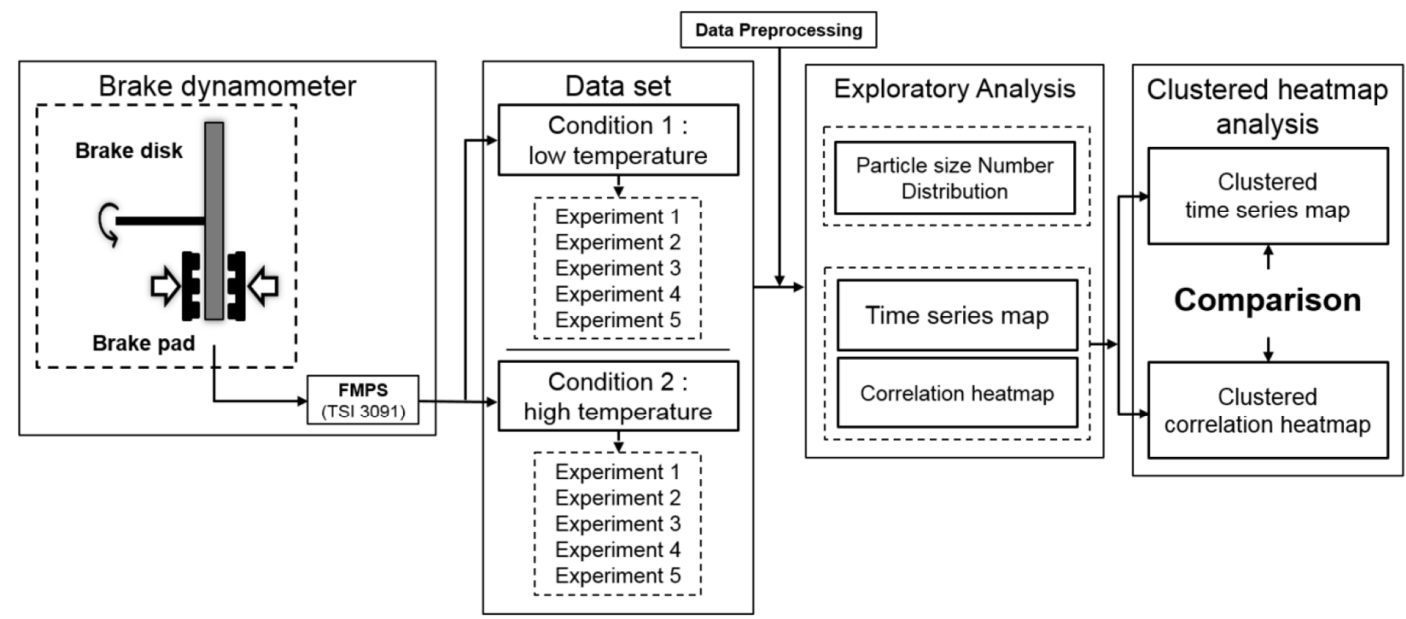

Fig. 1. Conceptual work flow of this research. 
Table 1. Changes in Braking Conditions (Velocity, Temperature, Coefficient of Friction, and Breaking Force $\left[\mathrm{F}_{\mathrm{B}}\right]$ ) during the Braking Period. The Numbers Reported Denote the Mean Value of Each Parameter among Five Replicate Experiments

\begin{tabular}{|c|c|c|c|c|c|c|c|c|}
\hline \multirow{2}{*}{$\begin{array}{c}\text { Time } \\
\text { (s) }\end{array}$} & \multicolumn{4}{|c|}{ Low initial temperature conditions $\left(35^{\circ} \mathrm{C}\right)$} & \multicolumn{4}{|c|}{ High initial temperature conditions $\left(60^{\circ} \mathrm{C}\right)$} \\
\hline & Velocity $(\mathbf{k m} / \mathbf{h})$ & Temp $\left({ }^{\circ} \mathrm{C}\right)$ & Friction Coeff. & $\mathrm{F}_{\mathrm{B}}(\mathrm{kN})$ & Velocity $(\mathbf{k m} / \mathbf{h})$ & Temp $\left({ }^{\circ} \mathrm{C}\right)$ & Friction Coeff. & $\mathrm{F}_{\mathrm{B}}(\mathbf{k N})$ \\
\hline 1 & 58.13 & 39.35 & 0.30 & 0.88 & 58.37 & 64.02 & 0.01 & 0.27 \\
\hline 2 & 57.51 & 38.29 & 0.31 & 2.20 & 57.76 & 64.29 & 0.31 & 2.01 \\
\hline 3 & 56.70 & 38.54 & 0.33 & 3.52 & 57.14 & 64.29 & 0.35 & 3.15 \\
\hline 4 & 55.26 & 38.79 & 0.34 & 6.95 & 55.51 & 64.81 & 0.38 & 6.39 \\
\hline 5 & 54.04 & 39.05 & 0.36 & 7.84 & 54.08 & 64.81 & 0.38 & 7.88 \\
\hline 6 & 52.60 & 39.30 & 0.36 & 7.94 & 52.65 & 65.86 & 0.40 & 7.89 \\
\hline 7 & 51.58 & 40.08 & 0.36 & 8.03 & 50.82 & 66.65 & 0.41 & 7.89 \\
\hline 8 & 49.12 & 41.12 & 0.37 & 8.04 & 48.57 & 67.70 & 0.41 & 8.08 \\
\hline 9 & 47.69 & 41.89 & 0.37 & 8.05 & 46.73 & 69.01 & 0.41 & 8.08 \\
\hline 10 & 46.26 & 42.41 & 0.37 & 8.05 & 45.51 & 70.06 & 0.42 & 8.09 \\
\hline 11 & 44.82 & 43.45 & 0.38 & 8.57 & 43.88 & 71.11 & 0.42 & 8.09 \\
\hline 12 & 43.60 & 44.23 & 0.38 & 8.31 & 42.86 & 72.16 & 0.42 & 8.10 \\
\hline 13 & 42.37 & 44.74 & 0.38 & 8.31 & 40.82 & 72.94 & 0.42 & 8.11 \\
\hline 14 & 40.12 & 45.26 & 0.39 & 8.31 & 39.18 & 73.99 & 0.42 & 8.12 \\
\hline 15 & 38.48 & 46.04 & 0.39 & 8.31 & 37.14 & 74.78 & 0.42 & 8.12 \\
\hline 16 & 36.84 & 46.81 & 0.40 & 8.31 & 35.51 & 75.57 & 0.42 & 8.13 \\
\hline 17 & 35.82 & 47.33 & 0.40 & 8.31 & 34.29 & 76.09 & 0.43 & 8.13 \\
\hline 18 & 34.39 & 47.58 & 0.40 & 8.31 & 32.65 & 76.88 & 0.43 & 8.14 \\
\hline 19 & 31.52 & 48.36 & 0.40 & 8.31 & 30.82 & 77.67 & 0.43 & 8.15 \\
\hline 20 & 30.29 & 48.61 & 0.41 & 8.31 & 28.57 & 78.45 & 0.43 & 8.15 \\
\hline 21 & 28.66 & 49.13 & 0.41 & 8.31 & 26.12 & 78.45 & 0.42 & 8.07 \\
\hline 22 & 27.84 & 49.90 & 0.42 & 8.31 & 24.49 & 79.24 & 0.43 & 8.16 \\
\hline 23 & 26.40 & 49.90 & 0.42 & 8.31 & 22.65 & 79.24 & 0.43 & 8.17 \\
\hline 24 & 24.36 & 50.41 & 0.42 & 8.31 & 21.02 & 80.03 & 0.43 & 8.18 \\
\hline 25 & 21.90 & 51.18 & 0.43 & 8.31 & 20.00 & 80.55 & 0.43 & 8.10 \\
\hline 26 & 20.67 & 51.44 & 0.43 & 8.31 & 18.16 & 80.03 & 0.43 & 8.10 \\
\hline 27 & 19.04 & 51.43 & 0.43 & 8.31 & 16.94 & 80.82 & 0.43 & 8.20 \\
\hline 28 & 17.40 & 51.42 & 0.43 & 8.31 & 14.69 & 80.55 & 0.43 & 8.12 \\
\hline 29 & 15.56 & 51.93 & 0.42 & 8.31 & 12.86 & 81.08 & 0.44 & 8.13 \\
\hline 30 & 13.51 & 51.92 & 0.44 & 8.31 & 11.43 & 80.82 & 0.43 & 8.22 \\
\hline 31 & 12.49 & 52.18 & 0.44 & 8.31 & 10.20 & 81.60 & 0.44 & 8.22 \\
\hline 32 & 11.05 & 52.43 & 0.44 & 8.31 & 8.16 & 81.08 & 0.43 & 8.14 \\
\hline 33 & 9.42 & 52.68 & 0.44 & 8.31 & 6.53 & 81.60 & 0.45 & 8.24 \\
\hline 34 & 7.16 & 52.67 & 0.45 & 8.31 & 4.49 & 81.60 & 0.44 & 8.25 \\
\hline 35 & 5.12 & 52.66 & 0.44 & 8.31 & 3.06 & 81.60 & 0.46 & 8.25 \\
\hline 36 & 4.09 & 52.65 & 0.46 & 8.31 & 1.84 & 81.60 & 0.50 & 8.17 \\
\hline
\end{tabular}

preprocessing section. Second, a clustered heatmap requires a hierarchical clustering method. Third, a distance metric is needed to measure the space between clusters. Finally, an appropriate color scheme for the heatmap is required [22].

The current study used the pheatmap function [23] in the $\mathrm{R}$ programming language [24] to generate clustered heatmaps. The Ward's algorithm, whereby clusters are agglomerated and the increases in sum-of-square errors are minimized, was used for hierarchical clustering. This robust method featured by relative insensitivity to noise and outliers tends to cluster groups of similar sizes [25, 26]. The Euclidean metric was used as the distance metric, and the color scheme was RGB: red indicating higher values, and blue indicating lower values. The number of clusters was set at two to identify if the generated clusters generally coincide with the size classes (Table S2).

\subsubsection{Data analysis procedure}

To characterize the differences in particle emission patterns under low and high temperature conditions, particle size distributions, time series maps, and correlation heatmaps were analyzed. The 
analysis process was largely divided into exploratory and clustered heatmap analyses.

In the exploratory analysis, particle size distributions were analyzed to determine the peak channel of each temperature condition and test. A particle size distribution was plotted using the mean particle concentration that occurred during the braking period across the size channels, and the total particle concentrations were compared prior to plotting. The dataset that was not normalized was used to compare particle concentrations among the tests. In addition, to examine the interdependence between particle generation characteristics with braking time in each temperature condition and size channel, time series maps and correlation heatmaps were created. A time series map was a $36 \times 32$ heatmap that tiled the dataset according to the color scale, and the correlation heatmap was a $32 \times 32$ heatmap created from the size channel correlation matrix. In the clustered heatmap analysis, the hierarchical clustering approach was applied to the heatmap to generate the clustered time series map and the clustered correlation heatmap.

\section{Results and Discussion}

Different patterns among replicate tests may be due to the stochastic nature of brake wear particles. The stochasticity may arise from a combination of multiple factors, such as abrasion, adhesion, fatigue, delamination, and thermal decomposition, the effects of which all vary depending on brake wear mechanism conditions [18, 27-29]. Despite the differences, a common trend was obtained for each temperature condition, suggesting that temperature affects the particle generation patterns during the braking of railway vehicles.

\subsection{Particle Size Distributions}

Total particle number concentration generated from five tests in each of low and high temperature condition is listed in Table 2.
Table 2. Total Particle Concentrations under Low and High Temperatures

\begin{tabular}{|c|c|c|}
\hline \multirow[b]{2}{*}{ Experiment no. } & \multicolumn{2}{|c|}{ Total particle concentration (particles $/ \mathbf{c m}^{3}$ ) } \\
\hline & $\begin{array}{l}\text { Low temperature } \\
\text { condition }\left(35^{\circ} \mathrm{C}\right)\end{array}$ & $\begin{array}{l}\text { High temperature } \\
\text { condition }\left(60^{\circ} \mathrm{C}\right)\end{array}$ \\
\hline 1 & $3,376,3$ & $1,234,311(1.23 \mathrm{E}+6)$ \\
\hline lent 2 & $162,488(1.62 \mathrm{E}+5)$ & $1,622,406(1.62 \mathrm{E}+6)$ \\
\hline Experiment 3 & $2,631,165(2.63 \mathrm{E}+6)$ & $1,715,905(1.72 \mathrm{E}+6)$ \\
\hline hent 4 & $1,270,087(1.27 \mathrm{E}+6)$ & $1,678,243(1.68 \mathrm{E}+6)$ \\
\hline Experiment 5 & $1,519,469(1.52 \mathrm{E}+6)$ & $1,105,565(1.11 \mathrm{E}+6)$ \\
\hline
\end{tabular}

The variation in particle concentration among tests was greater under low temperature (ranging from 162,488 to $3,376,380$ particles $/ \mathrm{cm}^{3}$ ) than under high temperature (ranging from 1,105,565 to 1,715,9050 particles $/ \mathrm{cm}^{3}$ ). The lowest particle concentration generated under low temperature was about an order of magnitude smaller than the lowest concentration generated under high temperature.

Fig. 2 shows particle size distributions in low and high temperature conditions. The number of peaks for the distributions differed between two temperature conditions. Except for one case (test 2), low temperature conditions exhibited unimodal distributions, with the peak located within size channel 24, which belongs to the size class 2 . The bimodal shape was clearly seen in tests 1-4 under high temperature but was less evident in test 5 . One peak of the bimodal distributions under high temperature was found within size channels 23-24, similar to the peak location under low temperature. The other peak for the bimodal distributions was located at the smaller particle sizes, size channels 17-18, except for test 1 that exhibits a peak at size channel 12, which all belong to size class 1 .

\subsection{Temporal Patterns of Wear Particles}

Fig. 3 displays the distributions of particle concentration, under
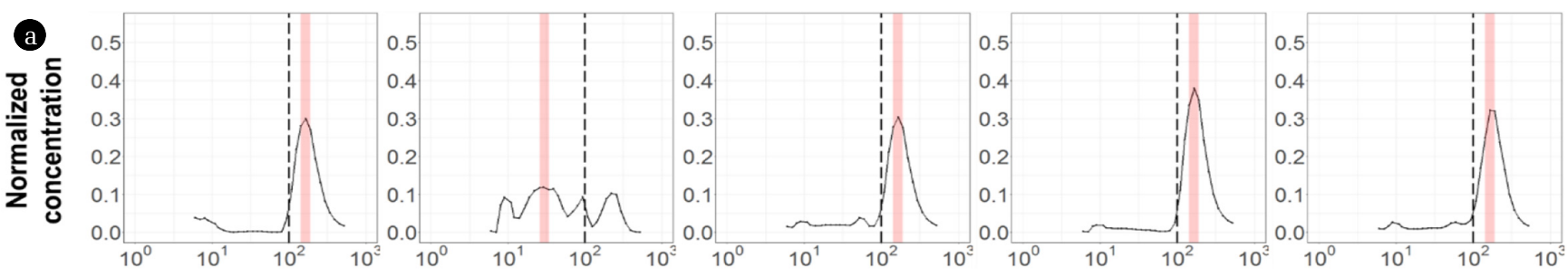

Particle size (nm)
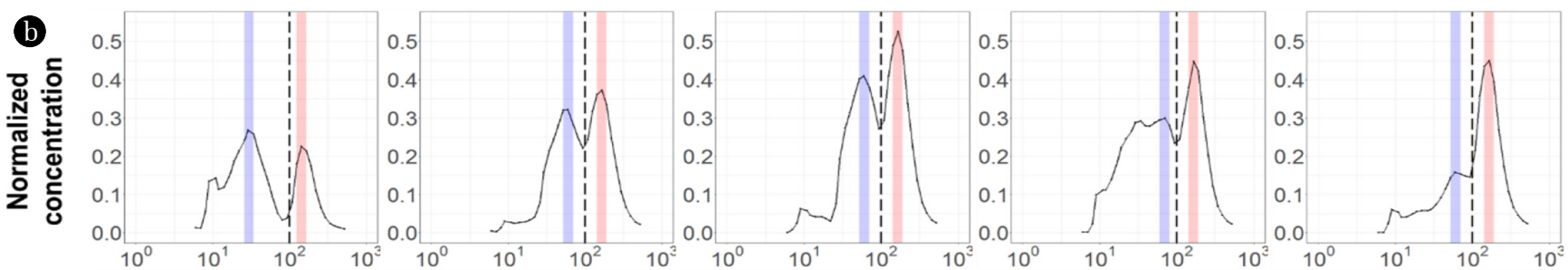

Particle size (nm)

Fig. 2. Normalized particle size number distributions under low temperature (a) and high temperature (b). The black vertical dashed line indicates a particle size of $0.1 \mu \mathrm{m}$, which is the cut-off point of the size class. Red and blue highlighted areas indicate the unimodal and bimodal peak of particle size number distributions under low temperature (a), and high temperature (b), respectively. 


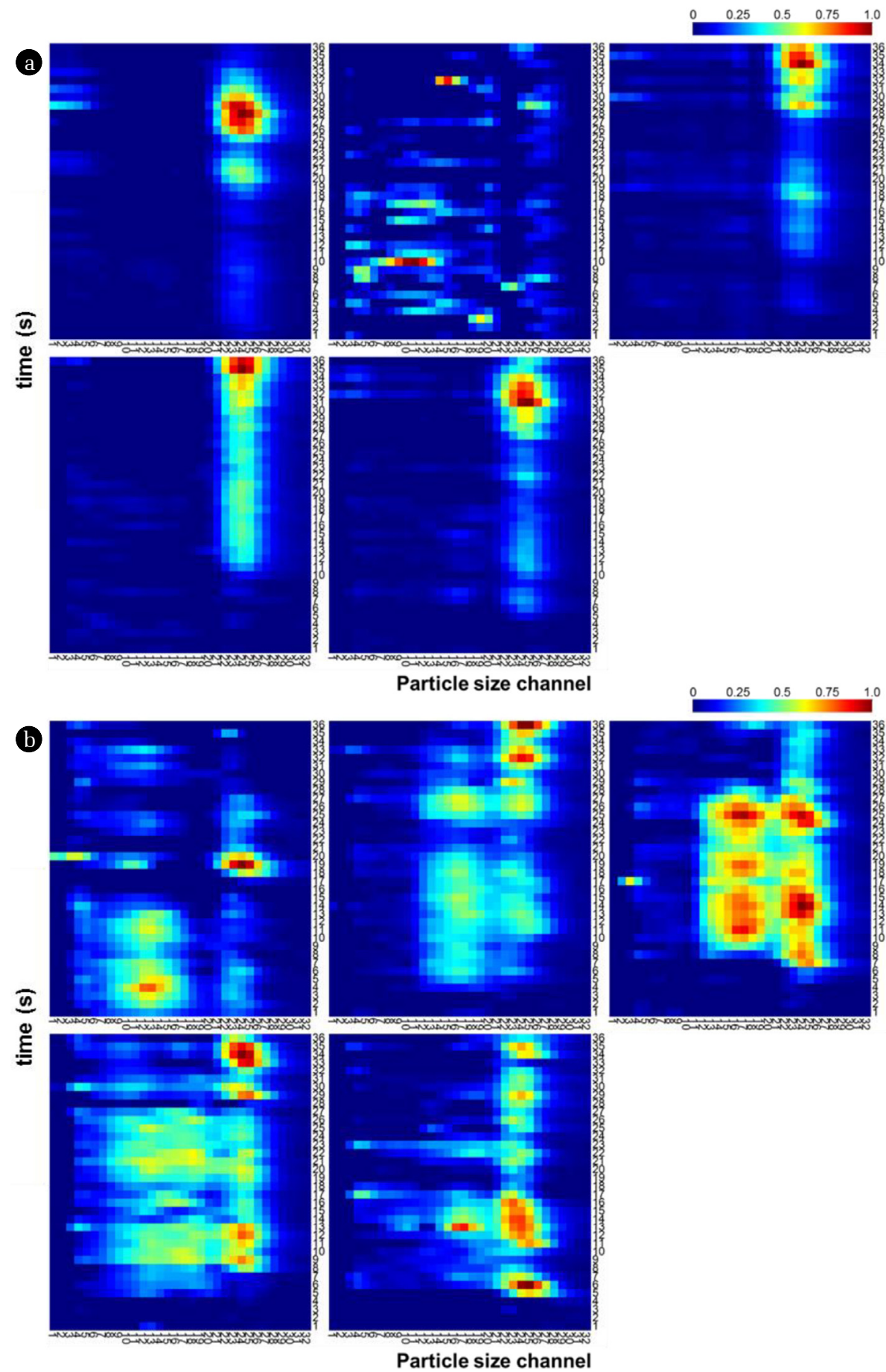

Fig. 3. Time series maps of normalized particle size with time series distribution, under low temperature (a) and high temperature (b). Each column represents particle size channels (1-32), while each row represents the braking time (1-36 s).

two different temperature conditions, in response to the changes in braking time and particle size test. The heatmap illustrates that under low temperature, particles with high concentration generally ranged between size channels 21-28, and occurred most frequently at 25-36 s, the latter end of the braking period (Fig. 3(a)). In contrast to the low temperature, at high temperature, the two peaks in terms of particle size occurred dispersedly along the braking period. Also, the peak timing was inconsistent among the replicate tests (Fig. 3(b)).

The clustered heatmap illustrates the hierarchical clustering 


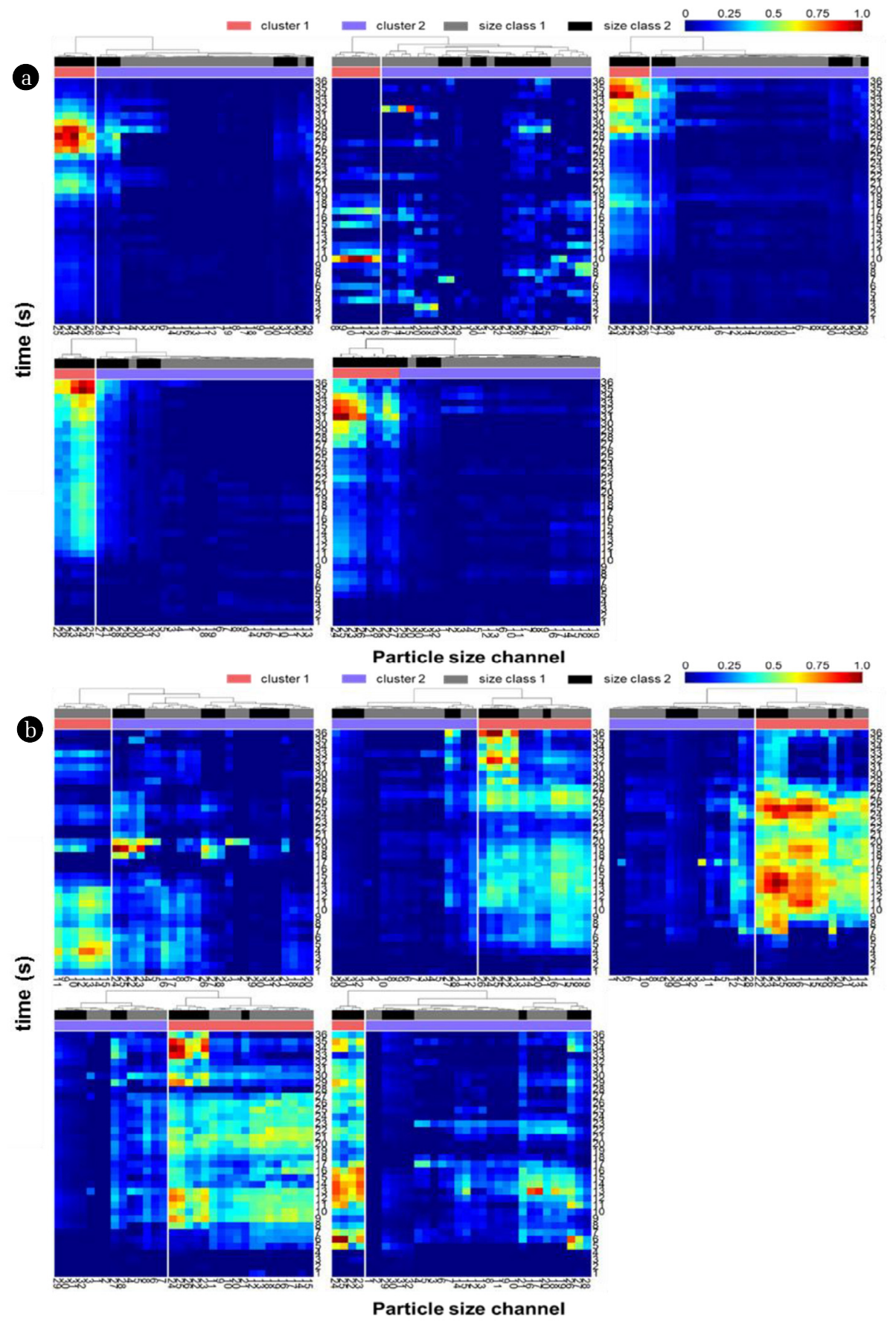

Fig. 4. Clustered time series maps of the normalized particle size with time series distribution under low temperature (a) and high temperature (b). Each row represents braking time (1-36 s), while each column represents the particle size channels (1-32). Size class 1 includes particle size channels 1-20, and size class 2 includes particle size channels 21-32, depicted with gray and black, respectively. Clusters 1 and 2 are annotated in light red and light blue, respectively.

results of the particle size channels for each time step along the braking time (Fig. 4). Clustering results in low temperature conditions demonstrate that there was one cluster (cluster 1) of channels with high particle concentrations, while the other cluster (cluster 2) contained channels with low particle concentrations. In the majority of the tests, the particle sizes with high occurrence frequency were, on average, larger than the particle sizes with low occurrence frequency throughout the braking period. The test 2 was an exception, in which small particles occurred more frequently than large particles did (Fig. 4(a)). As in the low temperature con- 


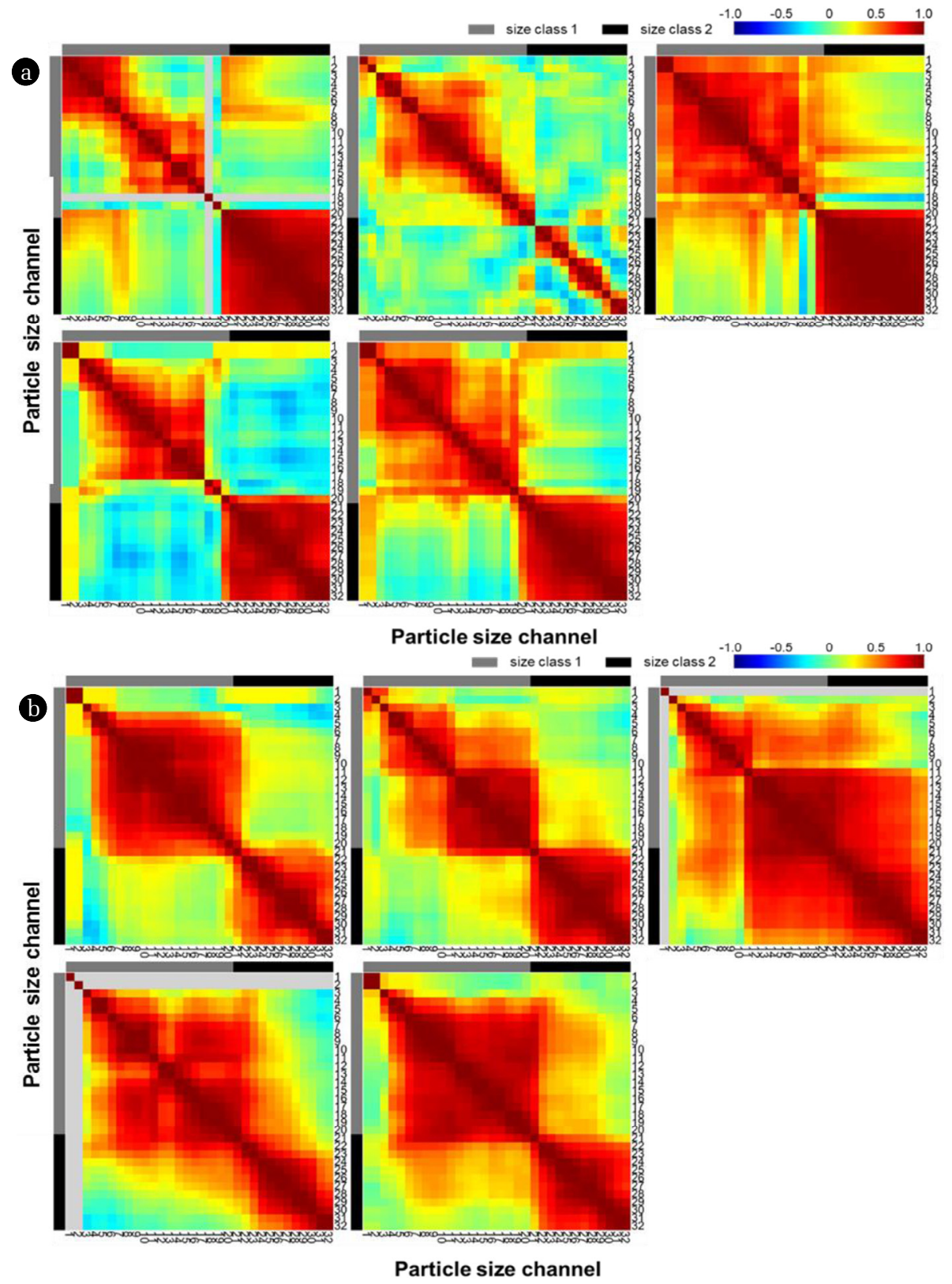

Fig. 5. Heatmaps of correlation matrix among particles across size channels 1-32, under low temperature (a) and high temperature (b). Size class 1 includes particle size channels 1-20 and size, while class 2 includes particle size channels 21-32, depicted in gray and black, respectively.

dition, under high temperature two clusters were generated in terms of occurrence frequency: cluster 1 with high particle concentrations and cluster 2 with low particle concentrations. In contrast to the results from low temperature, however, across the tests under high temperature, particle sizes in one cluster were not consistently larger or smaller than those in the other cluster (Fig. 4(b)).

\subsection{Correlations among Wear Particles}

Fig. 5 illustrates the correlation matrix among particles across size channels 1-32, under different temperature conditions. Regardless of the temperature, particles tended to covary with the similar sized particles. Given the low temperature, in particular, particles larger than approximately $80 \mathrm{~nm}$ showed a consistently high correlation to each other (Fig. 5(a)), with the Spearman's correlation coefficient close to one. Consistent with previous results (Fig. 2 - Fig. 4), the patterns derived from test 2 were dissimilar to other tests, without a clear sign of covariation among large particles. At high temperature, two groups of particles, 


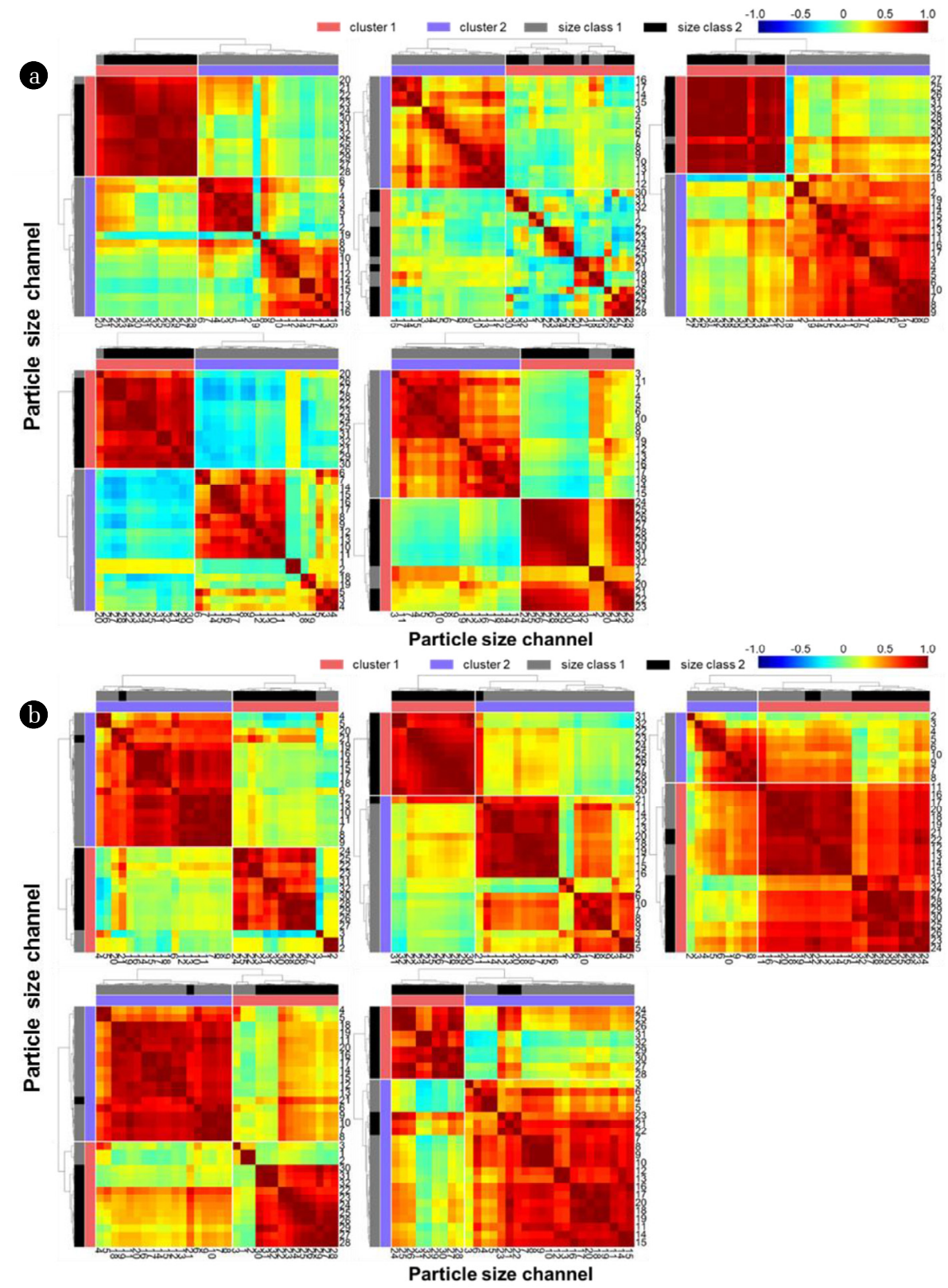

Fig. 6. Clustered correlation heatmaps among particles across size channels 1-32 under low temperature (a) and high temperature (b). Size class 1 includes particle size channels 1-20, while size class 2 includes particle size channels 21-32, depicted in gray and black, respectively. Clusters 1 and 2 are depicted in light red and light blue, respectively.

approximately in accordance with the size classes (except for test 3 at the smaller cutting point than size channel 21), were pronounced, and particles within each group were likely to vary together (Fig. 5(b)). However, the degree of correlation among the particles varied considerably within either group, and was inconsistent among the tests.

Fig. 6 is a clustered correlation heatmap created from the clustering results of the correlation matrix. Confirming the results in Fig. 5, the particles during braking were divided into two clusters with respect to particle size. Under low temperature, one cluster contained only the particles that belong to size class 1 , while the other cluster was generally associated with the particles that belong to size class 2 (Fig. 6(a)). Although the two clusters generated from high temperature represented particle size classification generally, they revealed greater heterogeneity compared to the clusters generated from low temperature.

\subsection{Comparison of Clustered Heatmaps}

Fig. S1 is the summary of particle size clustering with respect to the particle concentration along the braking time (Fig. S1(a) 
and (b)), and the degree of correlation among particles (Fig. S1(c) and (d)). The figure, unlike Fig. 4 or 6, informs the specific particle size channels that fall into each of the two clusters. Consistent with the unimodal distributions shown in Fig. 2(a), under low temperature, large particles between channels 21 and 28, within which a concentration peak was located, were classified as cluster 1, while other sized particles with lower concentrations were classified as cluster 2 (Fig. S1(a)). As shown in aforementioned results, the clustering pattern found in test 2 was different from that in other tests; smaller particles located between size channels 8-13 showed greater occurrence frequency, and were classified as cluster 1 . Under high temperature, the clustering results were comparable to the bimodal distributions shown in Fig. 2(b), with the particles between channels 9-26, where the dual concentration peaks were located, were classified as cluster 1 (Fig. S1(b)). However, the cluster 1 included only one of the dual peaks in two cases: test 1 , where dual concentration peaks were distant from each other, and test 5 , where the magnitude of the peak located at channel 17 was considerably weaker than that of the other peak. As shown in the correlation and clustered correlation heatmaps (Fig. 5 and 6), based on the degree of correlation, particles could be classified generally into two clusters, consisting of small and large sizes, respectively (Fig. S1(c) and (d)). Under low temperature, the cut-off point between cluster 1 and 2 was located at approximately channels 19-20, while under high temperature, the cut-off point was located in the majority of tests at approximately channels 20-21.

The clustered correlation heatmap results demonstrate that the particle size of approximately $100 \mathrm{~nm}$, the cut-off point between fine and ultrafine particles, also acted as the cut-off point which divides the particles into two groups in terms of particle generation characteristics, the timing of generation and concentration (Fig. 6, and Fig. S1(c) and (d)). This grouping would be in line with the finding that different mechanisms, mechanical wear and thermal decomposition, governed the generation of fine particles and ultrafine particles, respectively.

\subsection{Effects of Temperature on Brake Wear Particles}

Most noticeably, high temperature conditions promoted the generation of ultrafine particles. While one peak, located within the range of fine sized particles (most frequently at 143-165 nm), appeared for both low and high temperature, an additional peak, located within the range of ultrafine sized particles (most frequently at 60-70 nm), appeared only for high temperature (Fig. 2). The effect of high temperature on increasing ultrafine particle generation is in agreement with previous findings. Nosko et al. [14] found that $200^{\circ} \mathrm{C}$ is the critical temperature, above which ultrafine particles began to contribute to total particulate matters from car brake pads and discs. Similarly, Nosko et al. [13] reported that temperature increases shifted the dominant particle type from coarse to fine particles, and above about $170-190^{\circ} \mathrm{C}$ ultrafine particles became the dominant particle type. In Kukutchova et al. [12], increased generation of particles smaller than $100 \mathrm{~nm}$ was reported as average rotor temperature approached $300^{\circ} \mathrm{C}$. The disagreement of critical temperature among the studies may be due to the differing conditions and methods in which temperature is measured. Note that low and high temperature settings, $35^{\circ} \mathrm{C}$ and $60^{\circ} \mathrm{C}$, respectively, in our study represent the average temperature between the surface and inside of the disk at the initial stage of braking.

Under low temperature fine size particles were generated intensively at the latter end of the braking process (Fig. 3(a) and 4(a)), suggesting that particle concentration reached its peak as temperature approached the threshold, approximately $50^{\circ} \mathrm{C}$. Until the temperature reached and remained about $52.6^{\circ} \mathrm{C}$ at the end of braking, mechanical wear may dominate particle generation process, forming fine sized particles as the dominant particle type. In contrast, under high temperature the peaks for both fine and ultrafine particles were more spread out throughout braking (Fig. 3(b) and 4(b)). With the initial temperature of $60^{\circ} \mathrm{C}$, particles were actively generated from the early period of braking. The co-occurrence of fine and ultrafine particles indicates that together with mechanical wear, volatiles were generated from the thermal decomposition of less thermally stable components. These volatiles would undergo nucleation and coagulation to create smaller particles than the particles created from mechanical wear [12, $18,19,28,30]$.

\section{Conclusions}

Using heatmaps and clustered heatmaps, temperature effects on brake wear particles from railway vehicles were evaluated. The dataset was obtained by performing braking test, and five replicate tests was performed under low (initially $35^{\circ} \mathrm{C}$ ) and high (initially $60^{\circ} \mathrm{C}$ ) temperature conditions, respectively, to obtain generalized results. The study focused on characterizing the generation of nano-sized particles, rather than micro-sized particles, by measuring ultrafine (5.6-100 nm) and fine (100-560 nm) particles. The heatmap showed its capability to address multi-variable, time-series data with non-linear relationship, effectively capturing particle generation patterns along the braking period and size channel. Further, the clustered heatmap was able to create hierarchical groups, in which the particles with similar, generation characteristics, i.e., timing and concentration, were included, and the distance between groups revealed the degree of similarity.

\section{Acknowledgments}

This work was supported by the National Research Foundation of Korea (NRF) grant funded by the Korea government (MSIP) (No. NRF-2016R1C1B1014395).

\section{References}

1. WHO. Health effects of particulate matter. Policy implications for countries in eastern Europe, Caucasus and Central Asia. World Health Organization; 2013.

2. Abbasi S, Jansson A, Sellgren U, Olofsson U. Particle emissions from rail traffic: A literature review. Crit. Rev. Environ. Sci. Technol. 2013;43:2511-2544 
3. Amato F, Cassee FR, Denier van der Gon HAC, et al. Urban air quality: The challenge of traffic non-exhaust emissions. J. Hazard. Mater. 2014;275:31-36

4. Denier van der Gon HAC, Gerlofs-Nijland ME, Gehrig R, et al. The policy relevance of wear emissions from road transport, now and in the future - An international workshop report and consensus statement. J. Air Waste Manage. Assoc. 2013;63:136-149.

5. Harrison RM, Jones AM, Gietl J, Yin J, Green DC. Estimation of the contributions of brake dust, tire wear, and resuspension to nonexhaust traffic particles derived from atmospheric measurements. Environ. Sci. Technol. 2012;46:6523-6529.

6. Gasser M, Riediker M, Mueller L, et al. Toxic effects of brake wear particles on epithelial lung cells in vitro. Particle Fibre Toxicol. 2009;6:30.

7. von Uexküll O, Skerfving S, Doyle R, Braungart M. Antimony in brake pads-a carcinogenic component? J. Clean. Prod. 2005;13:19-31.

8. Garg BD, Cadle SH, Mulawa PA, Groblicki PJ, Laroo C, Parr GA. Brake wear particulate matter emissions. Environ. Sci. Technol. 2000;34:4463-4469.

9. Sanders PG, Xu N, Dalka TM, Maricq MM. Airborne brake wear debris: Size distributions, composition, and a comparison of dynamometer and vehicle tests. Environ. Sci. Technol. 2003;37:4060-4069.

10. Sioutas C, Delfino RJ, Singh M. Exposure assessment for atmospheric ultrafine particles (UFPs) and implications in epidemiologic research. Environ. Health Perspect. 2005;113:947-955.

11. Karlsson HL, Nilsson L, Möller L. Subway particles are more genotoxic than street particles and induce oxidative stress in cultured human lung cells. Chem. Res. Toxicol. 2005;18: 19-23.

12. Kukutschová J, Moravec P, Tomášek V, et al. On airborne nano/micro-sized wear particles released from low-metallic automotive brakes. Environ. Pollut. 2011;159:998-1006.

13. Nosko O, Alemani M, Olofsson U. Temperature effect on emission of airborne wear particles from car brakes. In: Europe's Braking Conference and Exhibition; 4-6 May 2015; Dresden.

14. Nosko O, Olofsson U. Quantification of ultrafine airborne particulate matter generated by the wear of car brake materials. Wear 2017;374:92-96.

15. Riediker M, Gasser M, Perrenoud A, Gehr P, Rothen-Rutishauser B. A system to test the toxicity of brake wear particle. Am.
J. Respir. Crit. Care Med. 2008;177.

16. Wahlström J, Söderberg A, Olander L, Jansson A, Olofsson U. A pin-on-disc simulation of airborne wear particles from disc brakes. Wear 2010;268:763-769.

17. Wahlström J, Olander L, Olofsson U. A pin-on-disc study focusing on how different load levels affect the concentration and size distribution of airborne wear particles from the disc brake materials. Tribol. Lett. 2012;46:195-204.

18. Kukutschová J, Filip P. Chapter 6 - A review of brake emission measurement studies: Identification of gaps and future needs. In: Amato F, 1st ed. Non-Exhaust Emissions: An Urban Air Quality Problem for Public Health; Impact and Mitigation Measures. Barcelona: Academic Press; 2018. p. 123-146.

19. Namgung H, Kim J, Woo S, et al. Generation of nanoparticles from friction between railway brake disks and pads. Environ. Sci. Technol. 2016;50:3453-3461.

20. Wilkinson L, Friendly M. The history of the cluster heat map. Am. Stat. 2009;63:179-184.

21. UIC code. 541-3 Brakes - Disk brakes and their application - General conditions for the approval of brake pads. 7th ed. Union Internationale des Chemins de Fer; 2010.

22. Weinstein JN. Biochemistry. A postgenomic visual icon. Science 2008;319:1772-1773.

23. Kolde R. Pheatmap-package: Pretty heatmaps. R package 2015.

24. Team RC. R: A language and environment for statistical computing [Internet]. Vienna, Austria: R Foundation for Statistical Computing; 2016;3:1-201.

25. Murtagh F, Legendre P. Ward's hierarchical agglomerative clustering method: Which algorithms implement ward's criterion? J. Classif. 2014;31:274-295.

26. Rencher AC, Christensen WF. Methods of multivariate analysis. 2nd ed. New York: John Wiley and Sons; 2003.

27. Aleksendrić D. Neural network prediction of brake friction materials wear. Wear 2010;268:117-125.

28. Talib R, Muchtar A, Azhari CH. Microstructural characteristics on the surface and subsurface of semimetallic automotive friction materials during braking process. J. Mater. Process Technol. 2003;140:694-699.

29. Xiao X, Yin Y, Bao J, Lu L, Feng X. Review on the friction and wear of brake materials. Adv. Mech. Eng. 2016;8:1-10.

30. Namgung H, Kim J, Kim M, et al. Size distribution analysis of airborne wear particles released by subway brake system. Wear 2017;372:169-176. 